\title{
A comparative study of bulk-fill composites: degree of conversion, post-gel shrinkage and cytotoxicity
}

\section{Alejandra ZUÑ̃IGA CISNEROS(a) Alessandra Sojo ARMAS(a)}

(a) Universidad Peruana de Ciencias Aplicadas, Escuela de Odontología Lima, Lima, Peru.

Declaration of Interests: The authors certify that they have no commercial or associative interest that represents a conflict of interest in connection with the manuscript.

\section{Corresponding Author:}

Alejandra Zuñiga Cisneros E-mail: u201414096@upc.edu.pe, morganaorange@gmail.com

hitps://doi.org/10.1590/1807-3107bor-2019.vol33.0003

Submitted: July 10, 2018

Accepted for publication: November 26, 2018

Last revision: December 04, 2018

\section{Dear Editor,}

We have reviewed the interesting article by Gonçalves et al. ${ }^{1}$ about the comparison of the degree of conversion, degree of contraction and cytotoxicity in Bulk Fill composites. We found the study of great importance for the clinical activity. It is remarkable that they have used six different Bulk Fill composites brands in different presentations considering their properties. However, we would like to comment on four aspects found that could be taken into account for future studies.

First, in the methodology they mention that all the samples were photopolymerized under the same parameters. As well, Bulk Fill composites presents a specific photoinitiator and this determines the power and time of light curing; because in each one the spectrum of light absorption differs. ${ }^{2}$ Therefore, it is important to follow the manufacturer's instructions, since the degree of conversion and contraction can vary. ${ }^{2}$

Also, it is not specified if the specimens were evaluated immediately after photopolymerization. It is relevant to know the detailed protocol in the elaboration of the specimens, as several studies mention that the photopolymerization continues up to 24 hours immersed in distilled water for 24 hours at $37^{\circ} .2,3,4$

On the other hand, to evaluate the volumetric contraction, a hemisphere was used considering the isotropic and homogeneous properties of the resins . A property of isotropy and homogeneity could be considered if the diameter of the light curing unit covers the whole hemisphere, since it influences the degree of conversion and the distribution of light in a homogeneous way. ${ }^{3)}$ If the tip does not cover the entire sample, there will be spaces that can not be measured, generating a slant in the results.

Finally, the inorganic particle size was considered the main cause of the degree of conversion. ${ }^{1}$ However, an adequate degree of conversion also depends on the light-curing conditions. ${ }^{2}$ It has been shown that the intensity of the lamp is reduced by absorption decreasing their potential. ${ }^{2}$ In addition, radiant energy and the level of radiation at the correct wavelength is an important factor for this. ${ }^{4}$

We hope that these recommendations contribute to enrich future related studies and to better understand the methodology and the different parameters that must be taken into account to evaluate the properties of Bulk Fill composites. 
A comparative study of bulk-fill composites: degree of conversion, post-gel shrinkage and cytotoxicity

\section{References}

1. Gonçalves F, Campos L, Rodrigues E, Costa F, Marques P, Francci CE et al. A comparative study of bulk-fill composites: degree of conversion, post-gel shrinkage and cytotoxicity. Braz Oral Res. 2018 Mar;32:e17. https://doi.org/10.1590/1807-3107bor-2018.vol32.0017

2. Gan KJ, Yap A, Cheong J, Arista N, Tan C. Bulk-fill composites: effectiveness of cure with poly- and monowave curing lights and modes. Oper Dent. 2018 Mar./ Apr;43(2):136-43. https://doi.org/10.2341/16-304-L
3. Shimokawa CAK, Turbino ML, Giannini M, Braga RR, Price RB. Effect of light curing units on the polymerization of bulk fill resin-based composites. Dent Mater J. 2018 Aug;34(8):1211-21. https://doi.org/10.1016/i.dental.2018.05.002

4. Karacolak G, Turkun L, Boyacioqlu H, Ferracane J. Influence of increment thickness on radiant energy and microhardness of bulk-fill resin composites. Dent Mater J. 2018 Mar;37(2):206-13. https://doi.org/10.4012/dmj.2017-032 